\title{
Energy input into the upper atmosphere associated with high-speed solar wind streams in 2005
}

\author{
Yue Deng, ${ }^{1}$ Yanshi Huang, ${ }^{1}$ Jiuhou Lei, ${ }^{2}$ Aaron J. Ridley, ${ }^{3}$ Ramon Lopez, ${ }^{1}$ \\ and Jeffrey Thayer ${ }^{2}$ \\ Received 11 October 2010; revised 25 March 2011; accepted 5 April 2011; published 4 May 2011.
}

[1] A 9 day periodic oscillation in solar wind properties, geomagnetic activity, and upper atmosphere has been reported for the year 2005. To understand the energy transfer processes from the high-speed solar wind streams into the upper atmosphere, we examined Joule heating and hemispheric power (HP) from the assimilative mapping of ionospheric electrodynamics (AMIE) outputs for 2005. There are clear 9 day period variations in all AMIE outputs, and the 9 day periodic oscillation in the global integrated Joule heating is presented for the first time. The band-pass filter centered at 9 day period shows that both Joule heating and HP variations are correlated very well to the neutral density variation. It indicates that the energy transfer process into the upper atmosphere associated with high-speed solar wind streams is a combination of Joule heating and particle precipitation, while Joule heating plays a dominant role. The sensitivities of Joule heating and HP to the solar wind speed are close to 0.40 and $0.15 \mathrm{GW} /(\mathrm{km} / \mathrm{s})$, respectively.

Citation: Deng, Y., Y. Huang, J. Lei, A. J. Ridley, R. Lopez, and J. Thayer (2011), Energy input into the upper atmosphere associated with high-speed solar wind streams in 2005, J. Geophys. Res., 116, A05303, doi:10.1029/2010JA016201.

\section{Introduction}

[2] During the declining phase of the solar cycle, when the IMF is decreasing and the solar irradiation remains almost constant [Gibson et al., 2009], high-speed solar wind streams (HSSs) are typical and dominate the Sun-Earth interaction. For much of 2005, an unusual arrangement of three equally spaced coronal holes resulted in a sequence of HSSs that corotated past the Earth at intervals of 9 days [Temmer et al., 2007]. The strong correlation between these solar wind stream, geomagnetic activity and thermosphere density at a 9 day period has been reported recently [Lei et al., 2008a]. Specifically, a 9 day recurrence seen in neutral densities derived from accelerometer measurements on the CHAMP satellite [Reigber et al., 2002] is simultaneously present in the solar wind and geomagnetic activity index Kp, but not in the solar EUV flux [Qian et al., 2010], which indicates a strong linkage between solar wind energy input into the magnetosphere and thermospheric density. Thayer et al. [2008] reported a predominant 7 day oscillation in the 2006 CHAMP-derived thermospheric density, and related this oscillation to solar wind fast streams. The global

\footnotetext{
${ }^{1}$ Department of Physics, University of Texas at Arlington, Arlington, Texas, USA.

${ }^{2}$ Department of Aerospace Engineering Sciences, University of Colorado at Boulder, Boulder, Colorado, USA.

${ }^{3}$ Center for Space Environment Modeling, University of Michigan, Ann Arbor, Michigan, USA.

Copyright 2011 by the American Geophysical Union. 0148-0227/11/2010JA016201
}

mean Total Electron Content (TEC) [Lei et al., 2008b] and $\Sigma \mathrm{O} / \mathrm{N}_{2}$ ratios measured by the Global Ultraviolet Imager (GUVI) on the TIMED satellite [Crowley et al., 2008] also present strong 9 and 7 day periodic oscillations in 2005 and 2006 , respectively, that are well correlated with the solar wind speed and $\mathrm{Kp}$ index.

[3] While it is proposed that the periodic oscillations observed in thermosphere/ionosphere are a direct response to recurrent geomagnetic activity and associated high-speed solar wind streams, the energy transfer processes between the solar wind, the magnetosphere and the ionosphere are not clear and still a key question for the solar wind/ magnetosphere/ionosphere coupling. The 9 day periodicity has been reported in the global electron particle precipitation [Emery et al., 2009; Zhang et al., 2010] and the localized Joule heating [Sojka et al., 2009], but no periodic oscillation in the global integrated Joule heating has yet been presented due to the difficulty of global measurement. At present, there are no direct ways to measure the total energy deposited by Joule heating in the polar region. It is however very important to confirm if the periodic oscillation exists in the integrated Joule heating and varies in the same manner as neutral density.

[4] In this study, we investigate two major magnetospheric energy deposition methods, Joule heating and particle precipitation, associated with this 9 day periodic oscillation. Joule heating and hemispheric power (HP) from assimilative mapping of ionospheric electrodynamics (AMIE) [Richmond and Kamide, 1988] output have been examined with the Lomb-Scargle spectral analysis. The correlation of the energy inputs to the neutral density variation has been 
evaluated using the band-pass filter centered at 9 day period. The sensitivities of Joule heating and HP to the solar wind speed have been examined as well.

[5] AMIE is a procedure to derive the realistic, timedependent, large-scale distribution of electromagnetic fields over the entire polar ionosphere by the synthesis of a variety of observations [Richmond and Kamide, 1988]. It is a form of optimally constrained, weighted least squares fit to all relevant data. There are many case studies for storm and substorm events by using AMIE outputs, while little effort has been made to study the Joule heating and other outputs for a long period, for instance, 1 year. In the present study, the Fuller-Rowell and Evans [1987] model and Weimer [1996] model have been used for the empirical conductance and electric potential, respectively. Only groundbased magnetometer data ( $>80$ magnetometers) for the entire year 2005 have been assimilated. The outputs of AMIE include Joule heating, HP, Dst, and cross polar cap potential (CPCP).

\section{Results and Discussion}

\subsection{Lomb-Scargle Analysis}

[6] The interaction between the high-speed stream (HSS) and the slower solar wind ahead of it forms a compression region, the corotating interaction region (CIR), in which the magnetic field intensity and solar wind density are enhanced due to the compression [Belcher and Davis, 1971]. When there is a strong 9 day periodic oscillation in solar wind, we expect that a similar oscillation appears in IMF. Figure 1 depicts the periodograms of solar wind speed and IMF intensity and components from Lomb-Scargle spectral analysis. The dashed lines are the upper limits of the $99 \%$ significance level. To reduce the effect of IMF sector polarity (Y. S. Kwak et al., Responses of thermospheric density to the interplanetary magnetic field sector polarity changes, submitted to Journal of Geophysical Research, 2010) and the high-frequency oscillation of the IMF in CIRs, we choose $\left|B_{x}\right|,\left|B_{y}\right|$ and $\left|B_{z}\right|$ over $B_{x}, B_{y}$ and $B_{z}$ in our analysis. The solar wind speed periodogram clearly shows a prominent peak at 9 days associated with the three equally spaced high-speed streams. There is also a 9 day peak in the IMF intensity and components that is related to the enhanced magnetic fields in the CIRs formed at the leading edges of the high-speed streams. Both IMF and solar wind velocity affect the magnetic reconnection rate in the magnetosphere, such as the dayside Magnetic Merging Rate $\left(M M R=V^{4 / 3} B_{T}^{2 / 3} \sin ^{8 / 3}\left(\theta_{c} / 2\right)\right.$ [Newell et al., 2008]), and the interplanetary electric field $(I E F=-V \times B)$, which directly controls magnetospheric energy inputs into the upper atmosphere. Therefore, there are two components associated to HSSs that influence the magnetospheric energy inputs into the upper atmosphere: the direct variation in solar wind velocity and the indirect variation in IMF. Since the effects of the variation in the solar wind speed and IMF cannot be separated through the data analysis in this study, the change of solar wind speed has been used to represent all the corresponding change in the solar wind conditions caused by HSSs.

[7] Figure 2 shows that the Lomb-Scargle periodograms of Joule heating, CPCP, HP and Dst index from AMIE output in 2005 have clear peaks at 9 days above the $99 \%$ significance level. Joule heating and particle precipitation are two primary methods through which the magnetospheric energy is deposited in the upper atmosphere. The 9 day periodicity in particle precipitation has been seen in NOAA particle observations [Emery et al., 2009] and TIMED GUVI observations [Zhang et al., 2010]. However, a 9 day periodicity of the global integrated Joule heating has not been reported due to the difficulty of measurement. The data assimilated model, AMIE, is an important method to estimate the variation of global Joule heating and other energy inputs, although it is limited by the distribution and quality of data and the assumptions carried by the model. For example, the distribution of the magnetometer data is not uniform and quite sparse at some locations. Joule heating related to the small-scale electric field variation is not well presented in the model. As shown in Figure 2, the existence of 9 day oscillation in the integrated Joule heating has been demonstrated for the first time. Comparison of the magnitudes of the 9 day oscillations between Joule heating and HP shows that the amplitude of Joule heating at 9 days is close to $30 \mathrm{GW}$ and almost two times as large as that of HP (14 GW), which indicates that Joule heating might be more important than HP in producing the 9 day oscillations in thermospheric density.

[8] The prominent feature of a 9 day periodicity in the solar wind speed during 2005 is quite unusual and different periodicity peaks may present in different years [Thayer et al., 2008]. Nevertheless, the coexistence of the 9 day periodic variation in both the solar wind speed and the magnetospheric energy inputs supports the cause and effect relationship between HSSs and thermospheric density enhancements. The ICMEs in 2005 [Richardson and Cane, 2010] produced significant geomagnetic storms and technically their contribution cannot totally be excluded in the processing. Since ICMEs are transient phenomena and have no well-organized repeatable feature with a 9 day periodicity like HSSs, their contribution to the 9 day period oscillations should not be dominant.

[9] Figure 1 shows a dominant 27 day peak in both solar wind speed and IMF conditions, which is directly related to the solar rotation. However, in Figure 2, there is no peak at 27 days but a primary peak at 30 days instead in both Joule heating and HP. The 30 day peak and 27 day minimum have also been shown in $\mathrm{Kp}$ [Lei et al., 2008a], HP and O/N2 from TIMED/GUVI observations [Zhang et al., 2010]. The stronger geospace driver in the 30 day component than in the 27 day component is a very interesting feature and will be investigated at some point.

\subsection{Band-Pass Filter and Sensitivity of Energy Inputs to the Solar Wind Speed}

[10] A band-pass filter centered at 9 days with range from 6 to 12 days has been utilized to further study the energy transfer processes from the high-speed solar wind streams into the upper atmosphere through Joule heating and particle precipitation. Figure 3 shows the percentage of the bandpass filtered Joule heating residuals (red) to the 12 day running mean and the percentage of the band-pass filtered CHAMP measured neutral density residuals (blue) to the 12 day running mean. The correlation coefficient between 

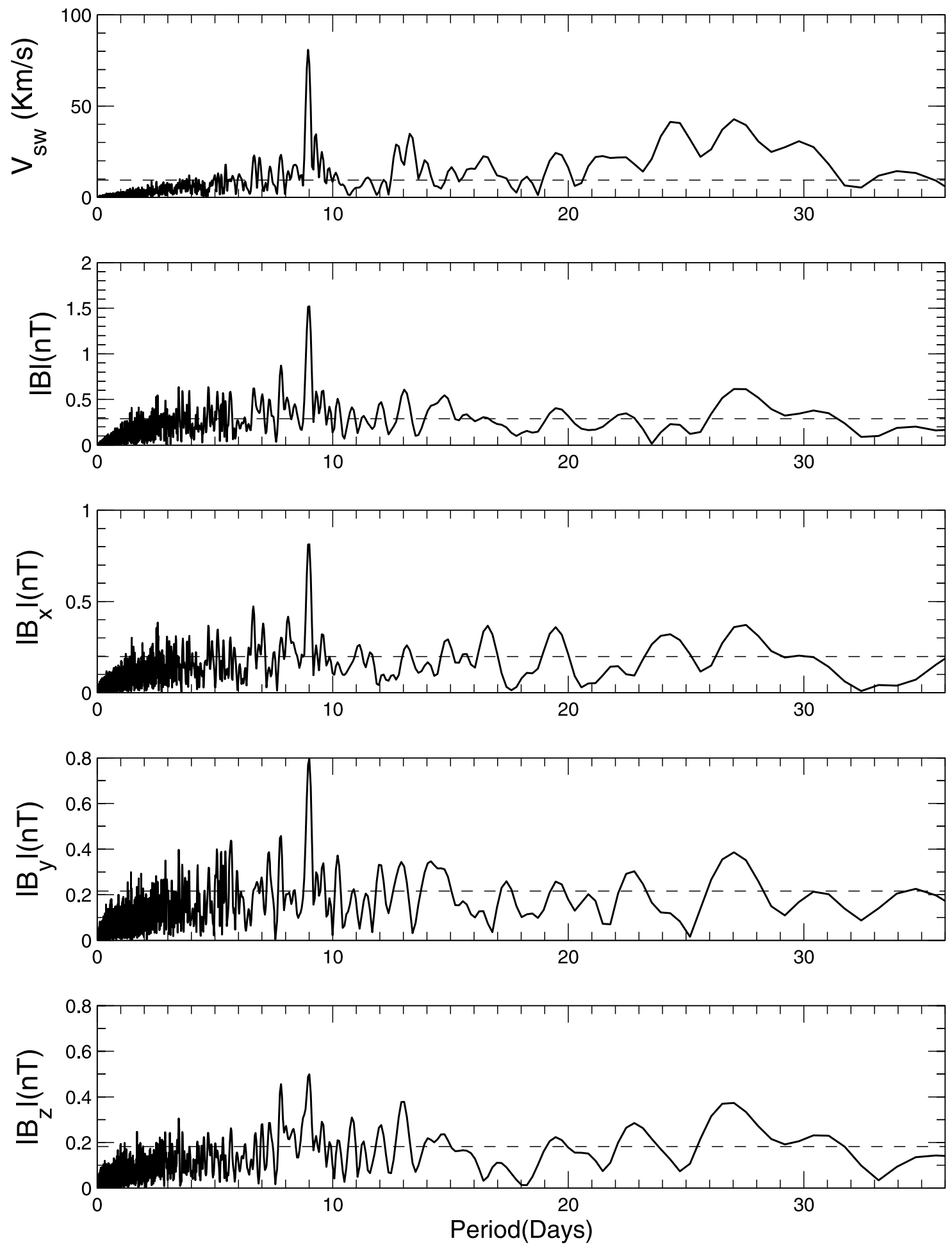

Figure 1. Periodograms of solar wind speed, $|B|,\left|B_{x}\right|,\left|B_{y}\right|$, and $\left|B_{z}\right|$ in 2005 from Lomb-Scargle spectral analysis (available at the Goddard Space Flight Center/Space Physics Data Facility OMNIWeb interface at http://omniweb.gsfc.nasa.gov). The dashed lines represent the $99 \%$ significance level.

Joule heating and neutral density is $r=0.93$. The correlation coefficient between HP and neutral density is also high $(r=0.92)$. The very good correlation between AMIE outputs and thermospheric neutral density for 6-12 days periodic oscillation suggests that the energy associated with the high-speed solar wind streams is dissipated in the upper atmosphere through both Joule heating and particle precipitation heating. 

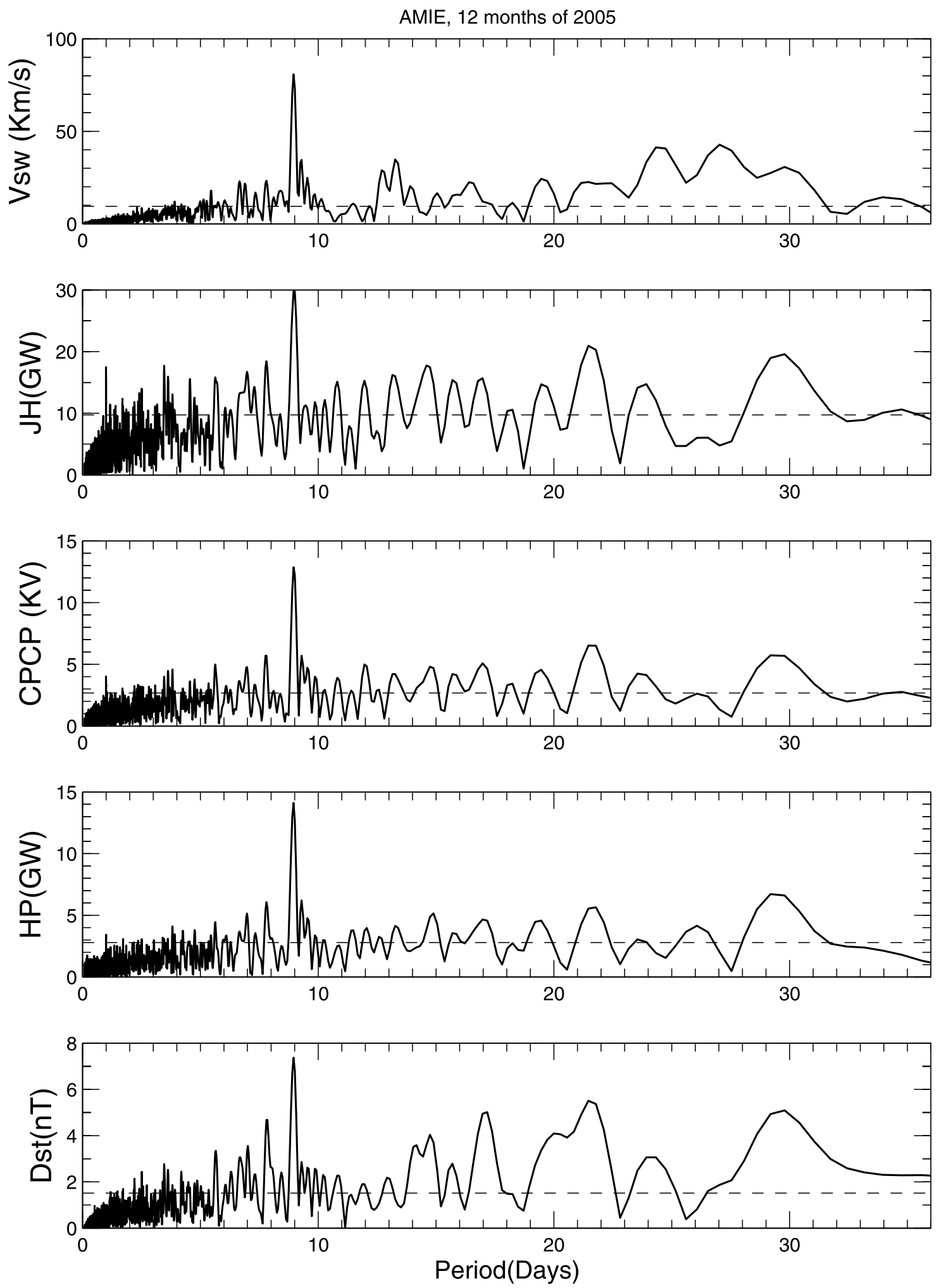

Figure 2. Periodograms of Joule heating, CPCP, hemispheric power, and Dst in 2005 from Lomb-Scargle spectral analysis of AMIE outputs. The dashed lines represent the $99 \%$ significance level.

[11] The sensitivities of Joule heating and HP variations to the solar wind speed change have been compared for the year 2005. The same band-pass filter process has been applied to the solar wind data. The band-pass filtered Joule heating residuals are then correlated with solar wind speed residuals after taking into account the time lag between them. Due to the nonsinusoidal nature of the periodicity, the specific period window (6-12 days) has been used for the 


\section{Band-pass filter centered at 9 days of AMIE outputs}
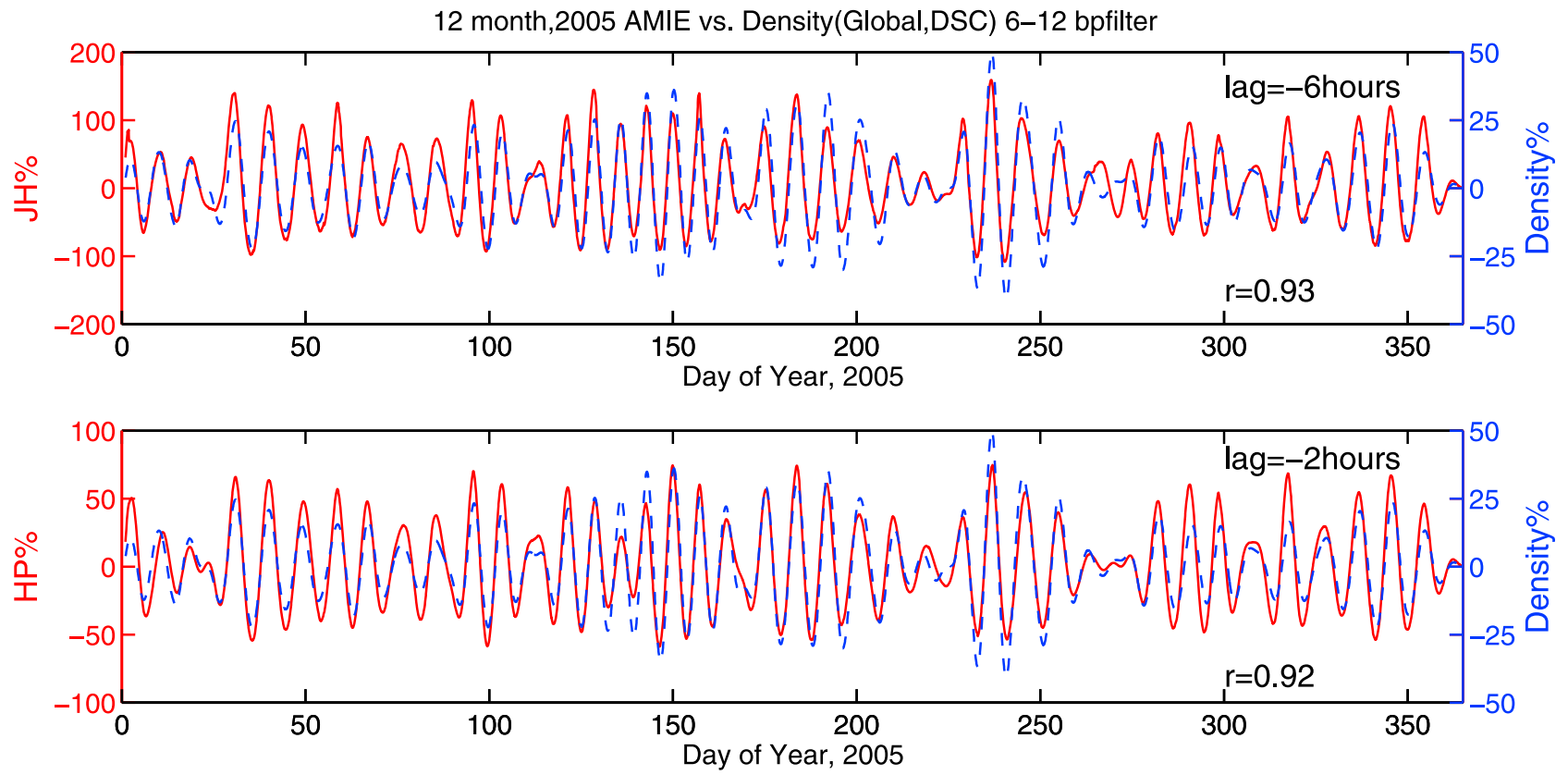

Figure 3. (top) The percentage of the band-pass filter Joule heating residuals (red) from AMIE output to the 12 day running mean and the percentage of the band-pass filter neutral density residuals (blue) from CHAMP satellite measurements to the 12 day running mean. The band-pass filter is centered at 9 days with range from 6 to 12 days. The $\mathrm{X}$ axis is the day of year in 2005, and the $\mathrm{Y}$ axis is the percentage difference. The correlation coefficient between Joule heating and neutral density is $r=0.93$, and the observed neutral density variation is $6 \mathrm{~h}$ after the Joule heating variation. (bottom) Same as Figure 3 (top) except for the HP and neutral density with $2 \mathrm{~h}$ lag between them.

Sensitivity of Joule heating to the solar wind velocity
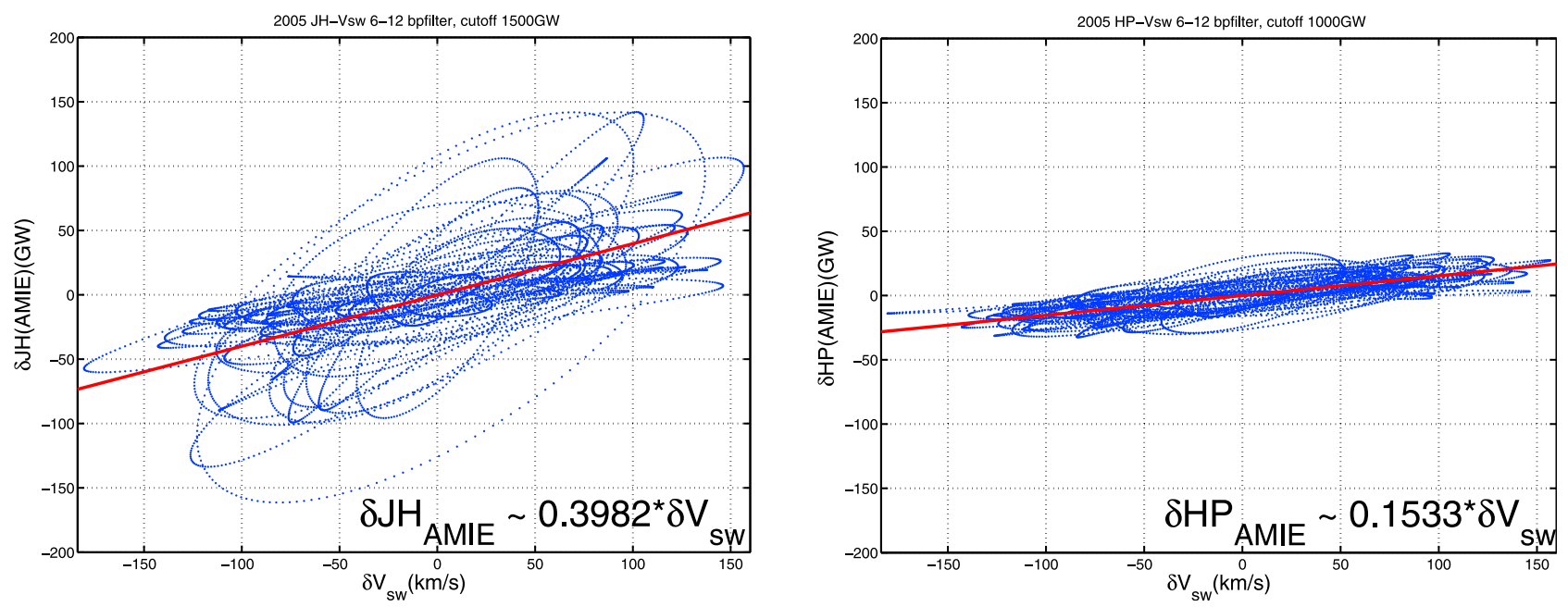

Figure 4. (left) Sensitivity of the Joule heating variation to the periodical oscillation of the solar wind speed. The $X$ axis is the solar wind speed residual after the 6-12 day band-pass filter, and the 0 point represents the average. The $\mathrm{Y}$ axis is the corresponding Joule heating residual after taking into account the time lag between Joule heating and solar wind speed. The linear fit is plotted out in the red line, and the sensitivity of the Joule heating to the solar wind speed is close to $0.40 \mathrm{GW} /(\mathrm{km} / \mathrm{s})$. (right) Same as Figure 4 (left) except for the sensitivity of hemispheric power (HP) to solar wind speed. The sensitivity of the HP to the solar wind speed is close to $0.15 \mathrm{GW} /(\mathrm{km} / \mathrm{s})$. 
band-pass filter. We have also investigated the situations with other window size (7-11 days and 8-10 days) and find only very slight change in the sensitivity $(\leq 5 \%)$.

[12] Figure 4 presents the variations of Joule heating and HP corresponding to the variation of solar wind speed. The red lines are the best linear fit, which show that the sensitivity of Joule heating to the solar wind speed is close to $0.40 \mathrm{GW} /(\mathrm{km} / \mathrm{s})$, and the sensitivity of HP to the solar wind speed is close to $0.15 \mathrm{GW} /(\mathrm{km} / \mathrm{s})$. When the solar wind changes from low-speed stream $(400 \mathrm{~km} / \mathrm{s})$ to highspeed stream $(700 \mathrm{~km} / \mathrm{s})$, Joule heating and HP increase by $120 \mathrm{GW}$ and $45 \mathrm{GW}$, respectively. In a quiet time, the average value of Joule heating and HP are close to $95 \mathrm{GW}$ and $36 \mathrm{GW}$ [Knipp et al., 2004]. These results indicate that the high-speed solar wind streams roughly double the quiet time magnetospheric energy inputs into the upper atmosphere and cause global variations. The red lines in Figure 4 only represent the statistical average. Due to the difference of the IMF and solar wind conditions other than the solar wind speed, the slope has a relatively broad range, including some outlying paths. Meanwhile, the red line may not represent the response to "pure" high-speed streams because of the influence of transient activities, but represents the yearly average response to high-speed streams.

[13] A direct comparison of the relative significance of Joule heating and HP on CHAMP densities should be made with caution, because Joule heating and HP influence the upper atmosphere through different processes. For HP, only a fraction ( $50 \%$ [Rees et al., 1983]) goes into heating the neutral atmosphere directly with the remainder going into ionization, dissociation and emission. In contrast, all of the Joule heating heats the atmosphere. Simulation study is needed in the future to compare the consequences in the thermosphere of the Joule heating and HP changes related to the high-speed solar wind streams. In this paper only the sensitivity of energy inputs to the solar wind speed has been presented. Given that the magnetospheric energy input into the upper atmosphere depends on both solar wind and IMF, the sensitivity to other parameters, such as $B V^{2}$ and $\rho V^{2}$, is also very important and has been investigated in a separate paper (Y. Huang et al., Comparison of Joule heating associated with high-speed solar wind between different models and observations, submitted to Journal of Atmospheric and Solar-Terrestrial Physics, 2011).

\section{Conclusion}

[14] The 9 day periodicity found in the upper atmosphere is consistent with a similar periodicity present in the solar wind speed. To understand the energy transfer processes into the upper atmosphere associated with high-speed solar wind stream, we first examined solar wind speed and IMF conditions in 2005 by Lomb-Scargle spectral analysis. The solar wind speed periodogram clearly shows a prominent peak at 9 days associated with the three equally spaced high-speed streams. There is also a 9 day peak in the IMF intensity and components that is related to the enhanced magnetic fields in the CIRs formed at the leading edges of the high-speed streams. The Lomb-Scargle periodograms of Joule heating, CPCP, HP and Dst index from AMIE outputs in 2005 have clear peaks at 9 days. The presence of 9 day periodic oscillation in integrated Joule heating is demonstrated for the first time.

[15] The strong correlation of the 9 day oscillation in Joule heating and particle precipitation to the variation in neutral density suggests that the energy transfer processes into the upper atmosphere associated with high-speed solar wind stream is a combination of Joule heating and particle precipitation, while Joule heating may play a more important role. The sensitivities of Joule heating and HP to the solar wind speed are close to $0.40 \mathrm{GW} /(\mathrm{km} / \mathrm{s})$ and $0.15 \mathrm{GW} /(\mathrm{km} / \mathrm{s})$. When the solar wind changes from lowspeed stream to high-speed stream, Joule heating and HP increase by $120 \mathrm{GW}$ and $45 \mathrm{GW}$, respectively, which indicates that the high-speed solar wind streams roughly double the quiet time magnetospheric energy inputs into the upper atmosphere and cause global variations.

[16] Acknowledgments. This research was supported by NSF grant ATM0955629. The work at CU was partly supported by the AFPSR MURI award FA9550-07-1-0565 and NASA grants NNX10AE62G and NNX10AQ52G.

[17] Robert Lysak thanks Ian Richardson and another reviewer for their assistance in evaluating this paper.

\section{References}

Belcher, J. W., and L. Davis Jr. (1971), Large-amplitude Alfvén waves in the interplanetary medium, 2, J. Geophys. Res., 76, 3534-3563, doi:10.1029/JA076i016p03534.

Crowley, G., A. Reynolds, J. P. Thayer, J. Lei, L. J. Paxton, A. B. Christensen, Y. Zhang, R. R. Meier, and D. J. Strickland (2008), Periodic modulations in thermospheric composition by solar wind high speed streams, Geophys. Res. Lett., 35, L21106, doi:10.1029/2008GL035745.

Emery, B. A., I. G. Richardson, D. S. Evans, and F. J. Rich (2009), Solar wind structure sources and periodicities of auroral electron power over three solar cycles, J. Atmos. Sol. Terr. Phys., 71, 1157-1175.

Fuller-Rowell, T. J., and D. Evans (1987), Height-integrated Pedersen and Hall conductivity patterns inferred from TIROS-NOAA satellite data, J. Geophys. Res., 92, 7606-7618.

Gibson, S. E., J. U. Kozyra, G. de Toma, B. A. Emery, T. Onsager, and B. J. Thompson (2009), If the Sun is so quiet, why is the Earth ringing?: A comparison of two solar minimum intervals, J. Geophys. Res., 114, A09105, doi:10.1029/2009JA014342.

Knipp, D. J., W. K. Tobiska, and B. A. Emery (2004), Direct and indirect thermospheric heating sources for solar cycles 21-23, Sol. Phys., 224, 495-505, doi:10.1007/s11207-005-6393-4.

Lei, J., J. P. Thayer, J. M. Forbes, E. K. Sutton, and R. S. Nerem (2008a), Rotating solar coronal holes and periodic modulation of the upper atmosphere, Geophys. Res. Lett., 35, L10109, doi:10.1029/2008GL033875.

Lei, J., J. P. Thayer, J. M. Forbes, Q. Wu, C. She, W. Wan, and W. Wang (2008b), Ionosphere response to solar wind high-speed streams, Geophys. Res. Lett., 35, L19105, doi:10.1029/2008GL035208.

Newell, P. T., T. Sotirelis, K. Liou, and F. J. Rich (2008), Pairs of solar wind-magnetosphere coupling functions: Combining a merging term with a viscous term works best, J. Geophys. Res., 113, A04218, doi:10.1029/2007JA012825.

Qian, L., S. C. Solomon, and M. G. Mlynczak (2010), Model simulation of thermospheric response to recurrent geomagnetic forcing, J. Geophys. Res., 115, A10301, doi:10.1029/2010JA015309.

Rees, M. H., B. A. Emery, R. G. Roble, and K. Stamnes (1983), Neutral and ion gas heating by auroral electron precipitation, J. Geophys. Res., $88,6289-6300$.

Reigber, C., H. Lühr, and P. Schwintzer (2002), CHAMP mission status and perspectives, Eos Trans. $A G U, 83(47)$, Fall Meet. Suppl., Abstract 307.

Richardson, I. G., and H. V. Cane (2010), Near-Earth interplanetary coronal mass ejections during solar cycle 23 (1996-2009): Catalog and summary of properties, Sol. Phys., 264, 189-237, doi:10.1007/s11207-010-9568-6.

Richmond, A. D., and Y. Kamide (1988), Mapping electrodynamic features of the high-latitude ionosphere from localized observations: Technique, J. Geophys. Res., 93, 5741-5759.

Sojka, J. J., R. L. McPherron, A. P. van Eyken, M. J. Nicolls, C. J. Heinselman, and J. D. Kelly (2009), Observations of ionospheric heating during the 
passage of solar coronal hole fast streams, Geophys. Res. Lett., 36 , L19105, doi:10.1029/2009GL039064.

Temmer, M., B. Vršnak, and A. M. Veronig (2007), Periodic appearance of coronal holes and the related variation of solar wind parameters, Sol. Phys., 241, 371-383.

Thayer, J. P., J. Lei, J. M. Forbes, E. K. Sutton, and R. S. Nerem (2008), Thermospheric density oscillations due to periodic solar wind high-speed streams, J. Geophys. Res., 113, A06307, doi:10.1029/2008JA013190.

Weimer, D. R. (1996), A flexible, IMF dependent model of high-latitude electric potential having "space weather" applications, Geophys. Res. Lett., 23, 2549-2552.
Zhang, Y., L. J. Paxton, and D. Morrison (2010), Auroral and thermospheric response to the 9 day periodic variations in the dayside reconnection rate in 2005, Space Weather, 8, S07001, doi:10.1029/2009SW000559.

Y. Deng, Y. Huang, and R. Lopez, Department of Physics, University of Texas at Arlington, 502 Yates St., Arlington, TX 76019, USA. (yuedeng@ uta.edu; yanshi.huang@mavs.uta.edu; lopez@uta.edu)

J. Lei and J. Thayer, Department of Aerospace Engineering Sciences, University of Colorado at Boulder, Boulder, CO 80309, USA. (jiuhou. lei@colorado.edu; jeffrey.thayer@colorado.edu)

A. J. Ridley, Center for Space Environment Modeling, University of Michigan, Ann Arbor, MI 48109, USA. (ridley@umich.edu) 